\title{
Evaluation of an Exercise Program for Diabetic Patients in Mongolia
}

\author{
Yasuhiro Endo ${ }^{1)}$, Chuluunbat Oyunchimeg ${ }^{2)}$, Kazumasa Nakagawa ${ }^{3)}$, Masaaki Sakamoto ${ }^{1)}$ \\ 1) Gunma University, Graduate School of Health Sciences: 3-39-22 Showa, Maebashi City, Gunma 371- \\ 8514,Japan.E-mail:yendo@health.gunma-u.ac.jp \\ 2) Health Sciences University of Mongolia \\ 3) Takasaki University of Health and Welfare
}

\begin{abstract}
Purpose] The purpose of this study was to validate the effects of an exercise program developed for healthy Japanese young men. This program was established as a basis for an exercise program for diabetic patients in Mongolia. [Methods] Ten healthy young men underwent the exercise tolerance test. They participated in a light exercise program consisting of 10 stretching exercises and 11 light exercises. During the exercise tolerance test and exercise program, heart rate (HR), oxygen uptake $\left(\mathrm{VO}_{2}\right)$, and rate of perceived exertion (RPE) were recorded. The intensity of the exercise program was determined by taking the average of the $\mathrm{VO}_{2}$ parameters during exercise and dividing it by the maximum value for $\mathrm{VO}_{2}\left(\mathrm{VO}_{2 \max }\right)$. Correlation coefficients for $\mathrm{VO}_{2}, \mathrm{HR}$, and $\mathrm{RPE}$ were calculated during the exercise tolerance test and all exercises. [Results] During the exercise program, $\mathrm{VO}_{2}$ was approximately $20 \%$ of $\mathrm{VO}_{2 \max }$, HR was $85-100 \mathrm{bpm}$, and RPE was 10-11. A high correlation coefficient was observed during the exercise tolerance test, and a moderate correlation was observed during the exercise program. [Conclusion] The intensity of the exercise program in this study was low to moderate. Thus, the exercise program must be revised after validating it among diabetic patients in Mongolia.

Key words: Exercise tolerance test, Exercise prescription, Lifestyle-related disease
\end{abstract}

(This article was submitted Sep. 6, 2012, and was accepted Oct. 21, 2012)

\section{INTRODUCTION}

The number of diabetic patients has increased substantially in many developing countries in recent decades because of changes in lifestyle and social environment resulting from rapid economic growth. This same tendency has been observed in Asian countries. For young people, researchers have advocated a preventative approach to counter lifestyle choices that may lead to type 2 diabetes ${ }^{1)}$. In 2011, the International Diabetes Federation estimated that 2.3 million people (age, 20-79 years) will be affected by diabetes in 20302), which is a rapid increase. In Mongolia, hypertension, diabetes, hypercholesterolemia and overweight are thought to be high cerebrovascular disease risk factors ${ }^{3}$. Approximately one-third of diabetes patients were diagnosed prior to the survey ${ }^{4}$, and $15.9 \%$ of all diabetics were adequately controlled ${ }^{3}$. Especially, obese people are increasing in Mongolia, and it was reported that one-third of the diabetic people were obese ${ }^{4}$ ). It was reported that there were proportionally more people in Mongolia than in Japan who were overweight or had a lot of body fat ${ }^{5}$ ). Ten years ago, prevention and management of diabetes were first established as important national goals for Mongolians ${ }^{4)}$

Diabetes is a preventable lifestyle-related disease. Exercise has been reported to be an effective means of decreasing blood sugar and controlling lipid metabolism, blood pressure, and weight. Exercise also enhances skeletal muscles, cardiopulmonary function, and psychological health; these benefits cannot be achieved by pharmacotherapy or dietary changes ${ }^{6,7)}$. In diabetic patients, enhanced cardiopulmonary function because of exercise has been linked with prolonged life ${ }^{8}$. The benefits of exercise in the prevention of diabetes are well known ${ }^{9,10)}$. Therefore, physiotherapists and other exercise professionals provide support for diabetes care and implement prevention programs.

In Mongolia, physiotherapy was first registered as a recognized profession in May 2011. The clinical reasoning and techniques that physiotherapists provide will be essential in Mongolia ${ }^{11)}$. Because physiotherapy is in the process of developing as a profession in Mongolia, effective exercise programs are currently lacking. Since the incidence of diabetes in Mongolia is increasing, effective exercise programs for diabetic patients must be devised and prescribed. Mongolian physiotherapists must play an active role in the treatment and prevention of diabetes.

We designed an exercise program in Japan called the Simple Exercise for Elderly People ${ }^{12}$, and we thought it might be suitable for Mongolia because it requires no special equipment. There are some differences (meals, culture, etc) between Japanese and Mongolians in their lifestyle, but there are also some common because of racial similarities. It was reported that proportion of people with metabolic abnormalities in Mongolia and Japan was not so different from Korea, but the proportion of people with of 


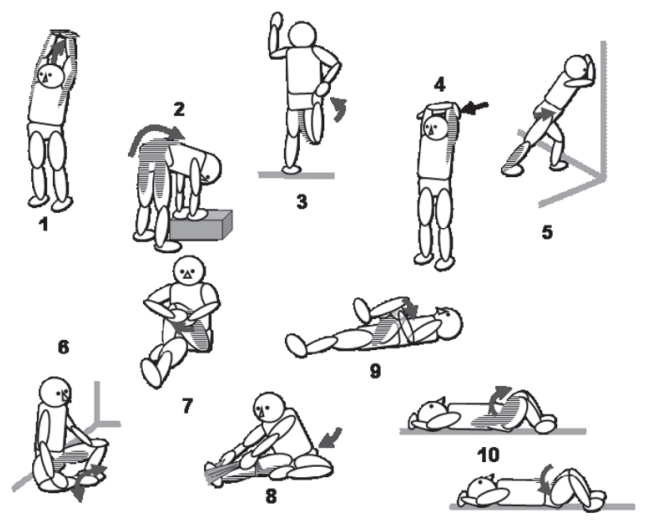

Fig. 1. Stretching exercises

Mongolian and Japanese people who do exercise more than twice a week was significantly less that of Korean people ${ }^{13)}$. Mongoloid populations were formerly considered to be relatively resistant to glucose intolerance ${ }^{4)}$. So, we thought it meaningful to validate an exercise program for Mongolian diabeties with Japanese in advance.

The purpose of this study was to validate the effects of an exercise program originally developed for healthy Japanese young men. And we believe that this result would help the physiotherapy for diabetes in Mongolia in future.

\section{SUBJECTS AND METHODS}

Ten healthy young Japanese men (age, $23.1 \pm 1.5$ years; height, $172.9 \pm 4.3 \mathrm{~cm}$; weight, $66.8 \pm 1.5 \mathrm{~kg}$ ) with no significant medical history were recruited for this study. All subjects were informed about the purpose and procedure of the study and provided their written informed consent to participation in advance.

An exercise tolerance test was performed by all subjects. The subjects then participated in a light exercise program. The exercise tolerance test used a cycle ergometer (AEROBIKE 75XLIII, Combi Wellness, Tokyo, Japan). It began at 20 watts and increased by 10 watts/min (ramp-type incremental exercise protocol) to the symptom-limited maximum. A 3 -min rest period (seated in the ergometer) and a 3-min warm-up preceded the test. The test was terminated when the cadence dropped to $<40$ revolutions/min, the subject requested termination of the test, or when the examiners judged that termination was best for safety reasons.

The light exercise program consisted of 10 stretching exercises and 11 light exercises, all of which can be easily performed at home. The required time was $20 \mathrm{~min}$ in total. A rubber tube and natural body weight were used in the resistance exercises. The stretching exercises of the exercise program are illustrated in Figure 1. Each stretch was performed twice for $20 \mathrm{~s}$. The light exercise program is illustrated in Figure 2. Exercises included sit-ups (10 repetitions), bridge (10 repetitions), straight leg raises (10 repetitions, both legs), lateral leg raises (10 repetitions, both legs), down on all fours with the opposite limbs raised (20 s,

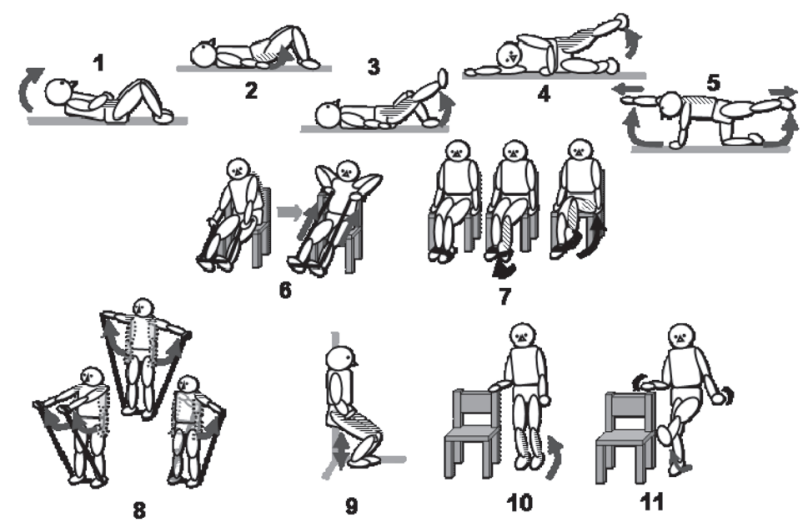

Fig. 2. Light exercises

(1) sit-up, (2) bridge, (3) straight leg raise, (4) lateral leg raise, (5) down on all fours with opposite limbs raised, (6) pull-up, (7) toe and thigh raise, (8) arm raise, (9) squat, (10) calf raise, (11) one-leg standing and leg swing

both sides), pull-ups (using an elastic tube; 10 repetitions), toe and thigh raises (using an elastic tube; 10 repetitions, both legs), arm raises (using an elastic tube; anterior, lateral, posterior, 5 repetitions per side), squats (10 repetitions), calf raises (20 repetitions), and one-leg standing and leg swinging (10 repetitions, both legs). All exercises were performed on a different day from the exercise tolerance test.

During the exercise tolerance test and light exercise program, heart rate $(\mathrm{HR})$, oxygen uptake $\left(\mathrm{VO}_{2}\right)$, carbon dioxide output per minute $\left(\mathrm{VCO}_{2}\right)$, and ventilatory equivalent (VE) were continuously recorded. HR was measured using a HR monitor (Polar FT1, Polar T31C transmitter; Polar Electro, Kempele, Finland). The other parameters were measured using an expiration gas analyzer (VO2000, S\&ME Inc., Nakano-ku, Tokyo, Japan) by the breathby-breath method. The anaerobic threshold (AT) was set at the following points: when $\mathrm{VCO}_{2} / \mathrm{min}\left(\mathrm{VCO}_{2}\right)$ and $\mathrm{VE}$ increased quickly, when $\mathrm{VCO}_{2}$ increased relative to $\mathrm{VO}_{2} /$ $\min \left(\mathrm{VO}_{2}\right)$, or at the point calculated by the $\mathrm{V}$-slope method. Maximum $\mathrm{VO}_{2}\left(\mathrm{VO}_{2 \max }\right)$ was calculated from the peak $\mathrm{VO}_{2}$ and $\mathrm{VO}_{2}$ at the AT point. Average $\mathrm{VO}_{2}, \mathrm{HR}$, and rate of perceived exertion (RPE, determined using the Borg scale) were measured during stretching and light exercise. During the exercise tolerance test, all parameters were measured at rest, 1-min intervals during exercise, and maximal exercise. During the light exercise program, all parameters were measured after completion of each exercise.

To examine the effects of the exercise program statistically, average $\mathrm{VO}_{2}$ values during stretching and light exercise were divided by $\mathrm{VO}_{2 \max }$ values. The resulting values were used to determine the intensity of the exercise program. Correlations among $\mathrm{VO}_{2}, \mathrm{HR}$, and RPE were calculated during the exercise tolerance test and all exercises using Spearman's rank correlation coefficient. SPSS version 16.0 for Windows (SPSS Inc., Chicago, IL, USA) was used for all calculations. 
Table 1. Results of $\mathrm{VO}_{2}, \mathrm{HR}$, and $\mathrm{RPE}$ at each point or period

\begin{tabular}{llccccc}
\hline & & Max & AT & Total & Stretching & Exercise \\
\hline \multirow{2}{*}{$\mathrm{VO}_{2} / \mathrm{min}$} & $\begin{array}{l}\text { Value } \\
(\mathrm{ml} / \mathrm{kg} / \mathrm{min})\end{array}$ & $42.1 \pm 0.6$ & $25.9 \pm 3.1$ & $8.7 \pm 1.5$ & $7.6 \pm 1.3$ & $9.7 \pm 2.0$ \\
\cline { 2 - 6 } & /Max (\%) & 100 & $61.5 \pm 7.2$ & $20.7 \pm 3.4$ & $18.0 \pm 2.9$ & $23.0 \pm 4.6$ \\
\hline \multirow{2}{*}{$\mathrm{HR}$} & $\begin{array}{l}\text { Value } \\
\text { (beats/minute) }\end{array}$ & & & $92.5 \pm 10.3$ & $85.7 \pm 6.4$ & $98.8 \pm 9.2$ \\
\hline $\mathrm{RPE}$ & Value & & & $10.0 \pm 2.0$ & $8.1 \pm 0.2$ & $11.7 \pm 0.3$ \\
\hline
\end{tabular}

Stretching: during stretching exercises; Exercise: during light exercises; Total: during all exercises. Values are expressed as means \pm standard deviations.

Table 2. Correlation coefficients among $\mathrm{VO}_{2} / \mathrm{min}, \mathrm{HR}$, and $\mathrm{RPE}(\mathrm{R})$

\begin{tabular}{cccccccc}
\hline & \multicolumn{3}{c}{ During exercise tolerance tests } & & \multicolumn{3}{c}{ during exercises } \\
\cline { 2 - 3 } \cline { 5 - 7 } \cline { 5 - 7 } $\mathrm{VO}_{2} / \mathrm{min}$ & $\mathrm{HR}$ & $\mathrm{RPE}$ & & $\mathrm{VO}_{2} /$ min & $\mathrm{HR}$ & $\mathrm{RPE}$ \\
\hline $\mathrm{VO}_{2} / \mathrm{min}$ & 0.841 & 0.842 & & & 0.496 & 0.466 \\
$\mathrm{HR}$ & & & 0.914 & & & 0.645 \\
$\mathrm{RPE}$ & & & & & & & \\
\hline
\end{tabular}

\section{RESULTS}

The average value of $\mathrm{VO}_{2 \max }$ was $42.1 \pm 0.6 \mathrm{~mL} / \mathrm{kg} / \mathrm{min}$. $\mathrm{VO}_{2}$ at the AT point was $25.9 \pm 3.1 \mathrm{~mL} / \mathrm{kg} / \mathrm{min}$, which was $61.5 \pm 7.2 \%$ of $\mathrm{VO}_{2 \max }$. During the stretching exercises, $\mathrm{VO}_{2}$ was $7.6 \pm 1.3 \mathrm{~mL} / \mathrm{kg} / \mathrm{min}\left(18.0 \pm 2.9 \%\right.$ of $\left.\mathrm{VO}_{2 \max }\right), \mathrm{HR}$ was $85.7 \pm 6.4 \mathrm{bpm}$, and RPE was $8.1 \pm 0.2$. During light exercise, $\mathrm{VO}_{2}$ was $9.7 \pm 2.0 \mathrm{~mL} / \mathrm{kg} / \mathrm{min}(23.0 \pm 4.6 \%$ of $\mathrm{VO}_{2 \max }$ ), HR was $98.8 \pm 9.2 \mathrm{bpm}$, and RPE was $11.7 \pm 0.3$. Over the entire exercise period, $\mathrm{VO}_{2}$ was $8.7 \pm 1.5 \mathrm{~mL} / \mathrm{kg} /$ $\min \left(20.7 \pm 3.4 \%\right.$ of $\left.\mathrm{VO}_{2 \max }\right)$, HR was $92.5 \pm 10.3 \mathrm{bpm}$, and RPE was $10.0 \pm 2.0$. All results for $\mathrm{VO}_{2}, \mathrm{HR}$, and $\mathrm{RPE}$ are shown in Table 1.

Correlation coefficient values during the exercise tolerance test were 0.841 between $\mathrm{VO}_{2}$ and $\mathrm{HR}, 0.842$ between $\mathrm{VO}_{2}$ and RPE, and 0.914 between HR and RPE. These values indicate high correlation. Correlation coefficient values during the exercise program were 0.496 between $\mathrm{VO}_{2}$ and $\mathrm{HR}, 0.466$ between $\mathrm{VO}_{2}$ and RPE, and 0.645 between HR and RPE. These values also indicate high correlation. All results of the correlation analysis are shown in Table 2.

\section{DISCUSSION}

$\mathrm{VO}_{2 \max }$ values for the study subjects were similar to the average values for young Japanese men reported in a previous study ${ }^{14)}$. The intensity of the exercise program in this study was low, as indicated by the $\mathrm{VO}_{2}$ values during the exercise program (approximately $20 \%$ of $\mathrm{VO}_{2 \max }$ ). It was reported that the effective exercise intensity for diabetes mellitus is from $50 \%$ to $60 \%$, or $30 \%$ to $40 \%$ for diabetic patients who do not exercise regularly ${ }^{15)}$. The exercise program in this study included exercises that are easy to implement, but individual tailoring of the intensity level was apparently insufficient to achieve adequate results. The program needs to be revised to increase the load during exercise. The program was developed considering that $\mathrm{VO}_{2 \max }$ of diabetic patients is most probably lower, and the intensity of the exercise program would be higher for them than for the study subjects. Because diabetic patients are more often middle aged, overweight, and less physically active, their $\mathrm{VO}_{2 \max }$ values are typically lower ${ }^{16)}$. Therefore, the exercise program in this study needs to be adapted for diabetic patients, and a revised version should be generated after validation among people with and at risk of diabetes in Mongolia. In this study, we designed the ramp slope as $10 \mathrm{watt} / \mathrm{min}$ on the basis of standards for diabetic patients ${ }^{15)}$, which is lower than the general indication. Because subjects in this study were healthy young men, we should have used a ramp slope of 20 to $30 \mathrm{watt} / \mathrm{min}^{15)}$. We think this explains why the intensity of the exercise program in this study was low.

The result of HR and RPE during light exercises shows that the exercises were moderate in intensity, which may be good for the target population. However, the effects of the program are still speculative because of the low correlation among the $\mathrm{VO}_{2}, \mathrm{HR}$, and $\mathrm{RPE}$ values. These results may be explained by the fact that many exercises in the program were effective not for the whole body but for specific muscles. Blood flow response to exercise for local muscles has been reported to differ from that to exercise for the whole body ${ }^{17)}$. Therefore, a more effective program would include exercises requiring use of the whole body.

In Mongolia, measurement of cardiopulmonary parameters such as $\mathrm{VO}_{2}$ may be difficult because of the lack of sufficient equipment. The high correlations among $\mathrm{VO}_{2}$, HR, and RPE in the exercise tolerance test demonstrate that HR and RPE measurement can be performed to determine the appropriate exercise intensity for diabetic patients in Mongolia.

\section{REFERENCES}

1) Chan JC, Malik V, Jia W, et al.: Diabetes in Asia. Epidemiology, risk factors, and pathophysiology. JAMA, 2009, 301: 2129-2140. [Medline] 
[CrossRef]

2) International Diabetes Federation: IDF Diabetes Atlas, 2011, 5th ed.

3) Otgontuya D, Oum S, Palam E, et al.: Individual-based primary prevention of cardiovascular disease in Cambodia and Mongolia: early identification and management of hypertension and diabetes mellitus. BMC Public Health, 2012, 12: 254. 3 .

4) Suvd J, Gerel B, Otgooloi H, et al.: Glucose intolerance and associated factors in Mongolia: results of a national survey. Diabet Med, 2002, 19 502-508. [Medline] [CrossRef]

5) Shiwaku K, Anuurad E, Enkhmaa B, et al.: Overweight Japanese with body mass indexes of 23.0-24.9 have higher risks for obesity-associated disorderes: a comparison of Japanese and Mongolians. Int J Obes, 2004 28: 152-158. [CrossRef]

6) Trovati M, Carta Q, Cavalot F, et al.: Influence of physical training on blood glucose control, glucose tolerance, insulin secretion, and insulin action in non-insulin-dependent diabetic patients. Diabetes Care, 1984, 7: 416-420. [Medline] [CrossRef]

7) Fluckey JD, Hickey MS, Brambrink JK, et al.: Effects of resistance exercise on glucose tolerance in normal and glucose-intolerant subjects. J Appl Physiol, 1994, 77: 1087-1092. [Medline]

8) Church TS, Cheng YJ, Earnest CP, et al.: Exercise capacity and body composition as predictors of mortality among men with diabetes. Diabetes Care, 2004, 27: 83-88. [Medline] [CrossRef]

9) Orozco LJ, Buchleitner AM, Gimenez-Perez G, et al.: Exercise or exercise and diet for preventing type 2 diabetes mellitus. Cochrane Database Syst Rev, 2008, 16: CD003054. [Medline]
10) Pan XR, Li GW, Hu YH, et al.: Effects of diet and exercise in preventing NIDDM in people with impaired glucose tolerance. Diabetes Care, 1997, 20: 537-544. [Medline] [CrossRef]

11) Nakazawa R, Sakamoto M, Oidov B: Support of physical therapy education in Mongolia. Kitakanto Med J, 2011, 61: 537-542 (in Japanese). [CrossRef]

12) Nakagawa $\mathrm{K}$, Inomata $\mathrm{N}$, Konno $\mathrm{Y}$, et al.: The characteristic of a simple exercise program under the instruction of physiotherapists -for general elderly people and frail elderly people-. J Phys Ther Sci, 2008, 20: 197-203. [CrossRef]

13) Shiwaku K, Nogi A, Kitajima K, et al.: Prevalence of the metabolic syndrome using the modified ATP 3 definitions for workers in Japan, Korea and Mongolia. J Occup Health, 2005, 47: 126-135. [Medline] [CrossRef]

14) The Office for Lifestyle-related Diseases Control, General Affairs Division, Health Service Bureau, Ministry of Health, Labour and Welfare of Japan: Exercise and Physical Activity Reference for Health Promotion 2006 (EPAR 2006). http://www0.nih.go.jp/eiken/programs/pdf/epar2006. pdf, (Accessed Aug 29, 2012).

15) American College of Sports Medicine: ACSM's Guidelines for Exercise Testing and Prescription, 8th ed. New York: Lippincott Williams \& Wilkins, 2009.

16) Siconolfi SF, Lasater TM, Snow RC, et al.: Self-reported physical activity compared with maximal oxygen uptake. Am J Epidemiol, 1985, 122: 101-105. [Medline]

17) Lind AR, McNicol GW: Cardiovascular responses to holding and carrying weights by hand and by shoulder harness. J Appl Physiol, 1968, 25: 261-267. [Medline] 